\title{
ARE C-REACTIVE PROTEIN LEVELS NECESSARY TO DIAGNOSE ACUTE APPENDICITIS IN CHILDREN?
}

\author{
J.D. Hodgkinson, Z.N. Shukur, E. Tan, O. Warren, A. Prabhudesai \\ Department of Colorectal Surgery, Hillingdon Hospital, London, UK
}

Background: Diagnosis of acute appendicitis is difficult, particularly in paediatric patients, where clinical signs maybe harder to elicit. Traditionally, history and clinical signs combined with white cell count (WCC) have been used in its diagnosis. Over recent years, C-reactive protein (CRP) is also being measured routinely.

Objective: To evaluate whether CRP improves diagnostic accuracy of acute appendicitis in children.

Population and methods: A retrospective study of 92 consecutive paediatric patients who underwent appendicectomy over a sixteen months $(\mathrm{M}=\mathrm{F}$; age $<16$ years). Inflammatory markers on presentation (CRP and WCC) were compared with histological findings.

Results: $65.2 \%$ (60) of patients had a histologically confirmed diagnosis of acute appendicitis. Use in isolation $\mathrm{WCC}>17$ yielded: sensitivity $=0.25$; specificity $=0.94$; $\mathrm{PPV}=0.88$. Used in isolation $\mathrm{CRP}>20$ yielded: sensitivity $=0.52$; specificity $=0.91$; PPV $=0.91$. Combined $\mathrm{WCC}>17$ and CRP $>20$ (sensitivity $=0.13$; specificity $=1.00 ;$ PPV $=1.00$ ) did not significantly improve PPV or specificity compared with WCC alone.

Conclusion: When used in isolation, WCC is more useful in predicting acute appendicitis at histology than CRP. Combining both markers has no impact on the likelihood of a correct diagnosis when compared with WCC $>17$ alone. Measuring CRP levels in suspected acute appendicitis does not improve diagnostic accuracy and therefore cannot be financially or clinically justified. 